\title{
Variability in Intense Tropical Cyclone Days in the Western North Pacific
}

\author{
Hirotaka Kamahori ${ }^{1}$, Nobuo Yamazaki ${ }^{1 *}$, Nobutaka Mannoji ${ }^{2}$, and Kiyotoshi Takahashi ${ }^{1}$ \\ ${ }^{1}$ Meteorological Research Institute/JMA, Tsukuba, Japan \\ ${ }^{2} J a p a n$ Meteorological Agency, Tokyo, Japan
}

\begin{abstract}
Variability in tropical cyclone (TC) days in the western North Pacific (WNP) since the late 1970s is investigated based on two datasets. As an overall behavior, the intense $\mathrm{TC}$ days have increased for the last 30 years from both the Japan Meteorological Agency (JMA) dataset and the Joint Typhoon Warning Center (JTWC) dataset. Both datasets show that TC days with an intensity of Saffir-Simpson category 2 or higher have increased by $15-30 \%$ over the past 30 years. In terms of the detailed behavior of this increase in intense TC days, the contribution obtained from the JMA dataset is different from that of the JTWC. The JMA dataset reveals that the increase in moderately intense TC days contributes to the overall increase in intense TC days, while the JTWC dataset shows that the increase in extremely intense TC days has the dominant contribution. The difference between the two datasets becomes significant after 1987 when aircraft reconnaissance by the US Air Force was deactivated. The difference between the assessed contributions is due to different implementations of the Dvorak technique, the basis for TC intensity estimation at the JMA and the JTWC after the deactivation of aircraft reconnaissance.
\end{abstract}

\section{Introduction}

In recent years, disasters due to TCs with maximum sustained winds of over 34kt have occurred frequently. The long-term variability of these TCs has become a social concern because of its relationship with climate change. In particular, TCs of category 4 or 5 on the Saffir-Simpson scale (Simpson 1974), with maximum sustained winds greater than $114 \mathrm{kt}$, are a potential cause of severe disasters, so it is important to investigate whether or not the frequency of these intense TCs has increased.

Webster et al. (2005) reports that the number of category 4 or 5 TCs has increased over global oceans, including the WNP, for the past 30 years. They suggest a relationship between the increase in extremely intense TCs and the sea surface temperature. Emanuel (2005) defines the power dissipation index (PDI), a temporal integration of the cubic power of the maximum sustained winds over their lifetime, as an index of potential TC disaster. He shows that the PDI has rapidly increased and that TCs have become more hazardous since the 1990s. Because the PDI is proportional to the cubic power of the maximum sustained wind, the contribution of extremely intense TCs is fairly large. The bulk of the PDI increase is due to the increasing frequency of extremely intense TCs. Goldenberg et al. (2001) found decadal scale hurricane activities in the northern

Corresponding author and present affiliation: Hirotaka Kamahori, Japan Meteorological Agency, 1-3-4 Ote-machi, Chiyoda-ku Tokyo, Japan. E-mail: kamahori@met.kishou.go.jp. *Present affiliation: Meteorological College. (C2006, the Meteorological Society of Japan.
Atlantic. They stress that the Atlantic basin was turned into an active period in hurricane activities after 1995. Landsea (2005) has demonstrated that the Atlantic basin tropical cyclone activity had similar heightened hurricane amounts in the middle portion of the 20th century in comparison to that observed since 1995. Landsea et al. (1999) found that only weak linear trends can be ascribed to hurricane activity in the north Atlantic. Chan and Liu (2004) point out that average typhoon activity had no significant relationship with sea surface temperature in the WNP. They also point out that the mean annual typhoon activity was generally higher (lower) during an El Nino (La Nina) year. This suggests a close relationship between typhoon activity and the ENSO. Chan (2006) insists that the increase in the occurrence of intense typhoons reported by Webster et al. (2005) was not a trend, but is a part of the large interdecadal variations in the number of intense typhoons, related to similar temporal fluctuations in the atmospheric environment.

In the WNP, the annual generation of TCs is about 30 , contributing about $30 \%$ of global generation (Neumann 1993). Considering TCs of typhoon or hurricane intensity defined by the World Meteorological Organization (WMO), with maximum sustained winds greater than or equal to $64 \mathrm{kt}$, the WNP accounts for $35 \%$ of global generation. Therefore, variations in TC intensity in the WNP play an important role in the global system.

The results of both Webster et al. (2005) and Emanuel (2005) for the WNP were based on the best track dataset from the Joint Typhoon Warning Center (JTWC). For the WNP, we can also use another dataset of TCs from the Japan Meteorological Agency (JMA). The JMA serves as the regional specialized meteorological center (RSMC) around the WNP for analyses and forecasts of TCs under the WMO. The JTWC serves mainly the US Air Force and the US Navy. Because both datasets were independently compiled for the same TCs in the WNP, it is important to verify whether similar results are obtainable with the JMA and JTWC datasets. We compared the TC intensities recorded in the two datasets.

In Section 2, outlines of the best track dataset from the JMA and that from the JTWC used in our analysis, are briefly described. In Section 3, the differences in TC intensity between the JMA and JTWC datasets, are quantitatively investigated. Conclusions and a discussion of the results are presented in Section 4.

\section{Dataset and method}

The datasets used are the best tracks of the JMA and the JTWC. Best track datasets are comprehensive compiled datasets of TC information, including their location, intensity, and size, taken at 6-hour intervals. All TCs after 1951 are recorded in the JMA best track. As for intensity information, it records the estimated central pressure over the whole period, but the estimated maximum sustained wind speed is recorded only 

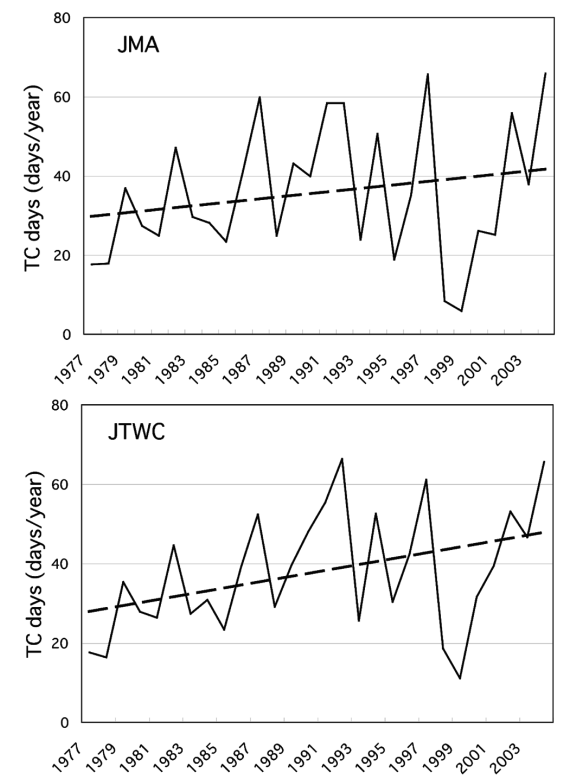

Fig. 1. Variability in annual TC days of category 2 or higher $(>=83 \mathrm{kt}$ ) in the WNP from the JMA best track (upper), and the JTWC best track (lower). Dashed lines indicate linear trends.

after 1977. All TCs after 1946 are recorded in the JTWC best track, with the maximum sustained wind speeds for all TCs represented in tables. There is no central pressure information for the whole period.

The main observational data sources used in TC analyses are aircrafts, satellites, surfaces, and upper air observations. Although the best tack datasets of both the JMA and the JTWC represent the same TCs and are based on almost the same observations, they are not the same. First, the JMA best track dataset records 10minute sustained wind speed as the maximum wind speed near the TC center following a recommendation by the WMO, while the JTWC uses 1-minute sustained wind speed. The strength of 10-minute sustained wind is statistically $88 \%$ of a 1 -minute sustained wind (Simiu and Scanlon 1978), resulting in a systematically weaker intensity of TCs in the JMA best track dataset. More importantly, the TC intensity cannot be directly observed, but are estimated by a combined analysis of available observations. The estimation methods used were developed independently by the JMA and JTWC. These methods led to different data for the same TCs in the JMA and the JTWC datasets. In 1987, an operational aircraft observation by the US Air Force, which was considered to be the most reliable observation to estimate TC intensity (Gray et al. 1991), was terminated in the WNP. Because aircraft observations are so accurate, we can expect them to bring very little ambiguity to the TC intensity estimation. We can thus expect consistent TC intensity information between the JMA and the JTWC datasets with aircraft observations. After 1987, a major source of TC intensity estimates has been the Dvorak technique, which handles satellite image patterns (Dvorak 1975). The supplement describes a history of a TC intensity estimation with the Dvorak technique at the JMA. Because the Dvorak technique includes a relatively larger ambiguity than the aircraft observations (Kossin and Velden 2004), it may introduce some differences in TC intensity estimation between the JMA and JTWC. By comparing the two best track datasets, therefore, we can expect to obtain more reliable information about the variability of TC intensity in the WNP basin.
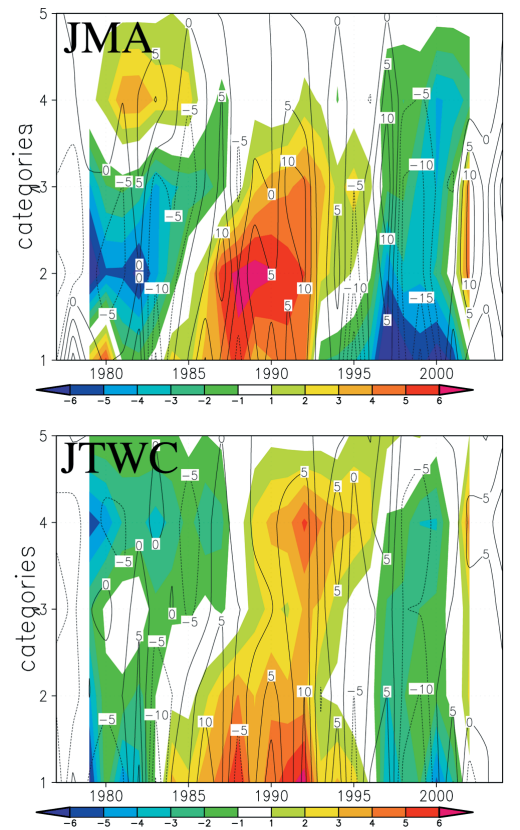

Fig. 2. Variability in anomaly of TC days in each category in the JMA (upper) and the JTWC (lower) datasets. Contour lines indicate the anomaly in each year and shading indicates a 5year running mean.

Atkinson and Holliday (1977) described frequent installations of algorithms for maximum wind estimation in the JTWC dataset before the early 1970s. To avoid this inhomogeneity in the JTWC dataset, we compared the variability of TC intensity in both datasets only for the period after 1977, when the maximum sustained wind was available in the JMA dataset and the algorithm differences had been minimized in the JTWC dataset.

There are two approaches to investigating the variability of TC intensity. One is to count the number of TC generations, and the other is to count TC days, integrating the lifetime of each TC along its path. We adopted the latter approach, because the degree of a TC disaster depends more strongly on the TC days. Therefore, our analysis of variability of TC intensity is influenced by the variability in their duration as well as the number of TCs generated.

As noted above, the maximum sustained wind speed is measured as a 10-minute average in the JMA best track dataset, while it is measured as a 1-minute average in the JTWC best track dataset. In order to compare the two datasets, we converted the maximum sustained wind speeds in the JMA best tack dataset to 1-minute averages by multiplying by $1.14(=1.0 / 0.88$, Simiu and Scanlon, 1978), except for the data used for Fig. 4.

\section{Variability in TC intensity}

Figure 1 shows the variability in the annual number of TC days of Saffir-Simpson category 2 or higher $(>=83 \mathrm{kt}$ ) in the WNP. Hereafter, a TC of category 2 or higher will be referred to as an "intense TC". There is a large year-to-year variability and a large decadal variability. However, both datasets reveal a qualitatively similar tendency for a definite increase of intense TC days since the late 1970 s.

Figure 2 depicts the variability of the anomaly in TC days in each category in the WNP averaged over the 
whole period. Both datasets showed quite similar decadal variability, highlighting the increase in the late 1980s through the early 1990s and the decrease in the late 1990s. We can also identify the difference between the JMA and JTWC. The JMA dataset shows a definite increase in moderately intense TC days, defined as TCs of category 2 or 3 , and a clear decrease in extremely intense TC days, defined as TCs of category 4 or 5 . On the other hand, the JTWC dataset indicates a significant increase in extremely intense TC days. Thus, the increases in intense (higher than category 2) TC days have different contributing factors in the JMA and JTWC datasets. Webster et al. (2005) concluded that the annual frequency of category 4 or higher TCs has rapidly increased in the WNP, based on the JTWC dataset. If the JMA dataset was used, a different conclusion, indicating a decreasing number of category 4 or higher TCs in the WNP, would be derived. In spite of these differences, a common increasing tendency can be seen in both datasets.

Table 1 lists the mean annual number of intense, moderately intense, and extremely intense TC days in the WNP for the periods 1977-1990 and 1991-2004. In the JMA dataset, intense TC days increased by $17 \%$ over the latter period. In contrast, the JTWC dataset shows a larger increase of $32 \%$, about twice the increase in the JMA dataset. For intense TC days, the two datasets indicate similar increasing trends. For moderately and extremely intense TC days, however, we found quite different tendency in the two datasets. The JMA dataset shows an increase in moderately intense TC days by $32 \%$ and a decrease in extremely intense TC days by $40 \%$ over the latter period. On the other hand, the JTWC dataset indicates an increase in extremely intense TC days by $72 \%$.

In order to explain why the two datasets have different contributions to the increase in the intense TC days seen in Fig.1, we compared TC days in both datasets. Figure 3 shows the difference between the TC days with moderately intense TCs and extremely intense TCs for the JMA and JTWC datasets. There are no significant differences between the JMA and the JTWC datasets for either moderately intense TC days or extremely intense TC days until 1987, when US Air Force reconnaissance was deactivated. After 1987, however, the annual number of moderately intense TC days in the JMA dataset became larger than those in the JTWC. In contrast, the number of extremely intense TC days in the JMA dataset became less than those in the JTWC dataset after 1987. Because TC intensity was estimated mainly with the Dvorak technique (Dvorak 1975) after the deactivation of aircraft reconnaissance, we have to consider that the Dvorak technique may be responsible for the different contributions in the two datasets.

We do not have sufficient evidence to judge which dataset is reasonable. To help us better understand the reliability of the datasets, we closely compared the two datasets. Figure 4 presents a histogram of TC days for each maximum sustained wind speed class before and after 1987, when reconnaissance by the US Air Force was deactivated. In Fig. 4, no conversion from the 10-minute

Table 1. Mean annual number of intense, moderately intense, and extremely intense TC days in the WNP for the periods 1977-1990 and 1991-2004.

\begin{tabular}{|c|c|c|c|c|c|c|}
\hline \multirow{2}{*}{} & \multicolumn{3}{|c|}{ JMA } & \multicolumn{3}{c|}{ JTWC } \\
\cline { 2 - 7 } & CAT & CAT & CAT & CAT & CAT & CAT \\
& $2-5$ & $2-3$ & $4-5$ & $2-5$ & $2-3$ & $4-5$ \\
\hline $1977-1990$ & 32.9 & 25.7 & 7.2 & 32.6 & 22.8 & 9.8 \\
\hline $1991-2004$ & 38.2 & 33.8 & 4.3 & 42.7 & 25.8 & 16.9 \\
\hline
\end{tabular}

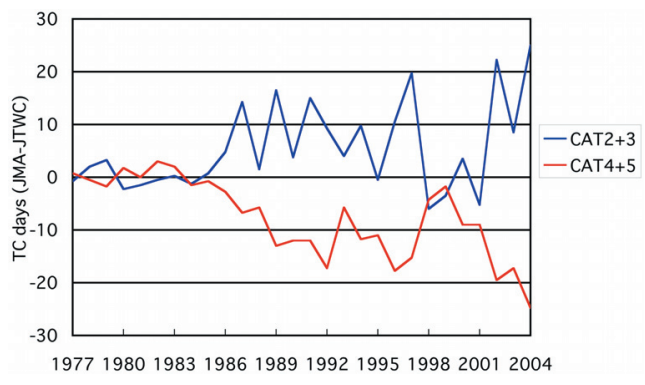

Fig. 3. Differences in TC days of category 2 or 3 (blue line) and category 4 or higher (red line) between the JMA and the JTWC datasets.
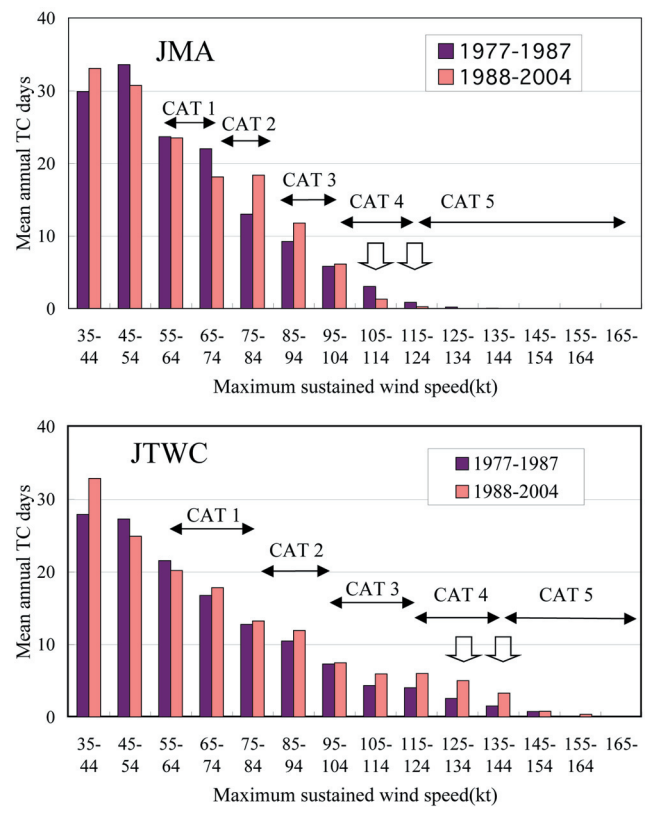

Fig. 4. Histogram of maximum sustained wind speed of TCs for period 1977-1987 and 1988-2004 from the JMA (upper) and the JTWC (lower). Horizontal arrows indicate each category range. Vertical arrows indicate significant difference between two periods with 95\% significant level in the t-test.

averaged wind speeds to 1 -minute wind speeds was made in the JMA dataset. Both datasets display a single peak distribution and a monotonic decrease in TC days with increasing maximum wind speed before 1987. After 1987, however, the two datasets show different trends. The JMA dataset shows an increase around the 75-94kt wind speed range, and a decrease around the 105-124kt range after 1987 . In contrast, the JTWC dataset shows an increase around the $115-144 \mathrm{kt}$ wind range. These variations are consistent with those in Fig. 2.

To examine data homogeneity between the two periods before and after 1987, we performed a t-test for each wind speed range. Significant differences were found between the two periods with a $95 \%$ significance level in the 105-114kt and 115-124kt ranges for the JMA dataset, and in the 125-134kt and $135-144 \mathrm{kt}$ ranges for the JTWC dataset, as indicated with vertical arrows in Fig. 4. There are two possible interpretations for the different results after 1987: one is due to climate change in TC intensity, and the other due to the change in the quality of source data for TC intensity estimation. It is possible that a climate change occurred around 1987. The two datasets, however, gave opposite results: the JTWC dataset shows an increase in extremely 
intense TC days, and the JMA dataset shows a decrease in extremely intense TC days and an increase in moderately intense TC days. A quality check of the two datasets has to be made before we attempt any physical interpretation of the changes in the intense TC days.

Because both datasets, although greatly different in quality, showed a similar increasing trend in TC days of category 2 or higher, we may expect that this is a statistically stable result.

We have seen that the JTWC and JMA sometimes assigned a different category for the same TC. This difference in TC assignment causes differences in the detailed changes in TC intensity. TC days of category 2 or higher appear to be less dependent on the detailed category assignment. The common increase in this intensity range gives a more robust conclusion, as reflected in Fig. 1.

\section{Conclusions and discussion}

We have obtained a consistent result that TC days of category 2 or higher (vmax $>=83 \mathrm{kt}$ ) have increased in the WNP basin over the past 30 years, based on datasets from both the JMA and JTWC. Because the datasets, which have largely different quality, show a similar increasing trend for TC days of category 2 or higher, we may expect that this is a statistically stable result. We will have to cope with the disasters brought by the expected increasing TC hazard in the WNP. Concerning which TC categories have contributed significantly to the increase in intense TC days, the JMA and JTWC datasets give different results. The JMA dataset shows an increase in moderately intense TC days with a SaffirSimpson category of 2 or 3 . On the other hand, the JTWC dataset indicates an increase in extremely intense TC days of category 4 or higher.

Emanuel (2005) and Webster et al. (2005) identified a definite increase in category 4 or higher TCs in the WNP after the 1990s, based on the JTWC dataset. If the JMA dataset is used, a different conclusion can be obtained, namely a definite decrease in category 4 or higher TCs. We also found that differences between the datasets were more significant after 1987. Until 1987, TC intensity estimation for both datasets was primarily based on the US Air Force reconnaissance. Because the quality of the aircraft observations was extremely high (Gray et al. 1991), we can expect that both datasets had very similar behavior before 1987, except for the difference in the averaging interval of maximum sustained wind speed. After deactivation of the US Air Force reconnaissance in 1987, the JMA and JTWC adopted the Dvorak technique to estimate the TC intensity in different ways. The JMA modified the original Dvorak technique to be consistent with surface observations near Japan (Osano 1989). In contrast, the JTWC adopted the original Dvorak technique, which was developed for north Atlantic hurricanes (Dvorak 1975). The different algorithms led to quantitatively different results for the same satellite imagery of the same TCs in the WNP.

Emanuel (2005) and Webster et al. (2005) discussed the relationship between an increase in category 4 or higher TCs and the sea surface temperature from the viewpoint of climate change. In the north Atlantic basin, the incidence of major hurricanes, defined as category 3 or higher, decreased during the 1970s and 1980s (Landsea 1993). Since the mid-1990s, north Atlantic hurricane activity has been in an increasing phase (Goldenberg et al. 2001). They found multidecadal variability of hurricane activity in the basin, and suggested that the present high level of hurricane activity is likely to persist for an additional 10 to 40 years. Yumoto and
Matsuura (2001) found decadal variability in the generation of TCs in the WNP basin, with peak phases in the 1960s and 1980s. It is certainly important to discuss the relationship between the variability of TC intensity and climate change. As a first step for such discussion, however, we need to verify the quality of the datasets, including the TC intensity information, because the available TC datasets have been compiled operationally and may be of temporally varying quality.

\section{Acknowledgments}

The authors would like to thank Mr. K. Kishimoto, A. Kikuchi, S. Yamada, and H. Koba of the JMA, and S. Nishimura of the Meteorological Satellite Center for valuable discussions. The authors also thank three reviewers for constructive comments.

\section{Supplement}

Chronological table of satellite analysis of TC intensity at the JMA.

\section{References}

Atkinson, G. D., and C. H. Holliday, 1977: Tropical cyclone minimum sea level pressure/ maximum sustained winds relationship for the western North Pacific. Mon. Wea. Rev., 105, 421-427.

Chan, J. C. L., and K. S. Liu, 2004: Global warming and western North Pacific typhoon activity from an observational perspective. J. Climate, 17, 4590-4602.

Chan, J. C. L., 2006: Comment on "Changes in tropical cyclone number, duration, and intensity in a warming environment". Science, 311, 1713b.

Dvorak, V. F., 1975: Tropical cyclone intensity analysis and forecasting from satellite imagery. Mon. Wea. Rev., 103, 420-430.

Emanuel, K., 2005: Increasing destructiveness of tropical cyclones over the past 30 years. Nature, 436, 686-688.

Goldenberg, S. B., C. W. Landsea, A. M. Mestas-Nunez, and W. M. Gray, 2001: The recent increase in Atlantic hurricane activity: Causes and implication. Science, 293, 474-478.

Gray, W. M., C. Neumann, and T. L. Tsui, 1991: Assessment of the role of aircraft reconnaissance on topical cyclone analysis and forecasting. Bull. Amer. Meteor. Soc., 72, 1867-1883.

Kossin, J. P., and C. S. Velden, 2004: A pronounced bias in tropical cyclone minimum sea level pressure estimation based on the Dvorak technique. Mon. Wea. Rev., 132, 165-173.

Landsea, C. W., 1993: Climatology of intense (or major) Atlantic hurricanes. Mon. Wea. Rev., 121, 1703-1713.

Landsea, C. W., R. A. Pielke, A. M. Mestas-Nunez, and J. A. Knaff, 1999: Atlantic basin hurricanes: Indices of climatic changes. Climatic Change, 42, 89-129.

Landsea, C. W., 2005: Hurricanes and global warming. Nature, 438, E11-13.

Neumann, C. J., 1993: Global overview. Global guide to tropical cyclone forecasting. World Meteorological Organization, Rep. TCP-31, WMO/TD-560, Geneva, Switzerland, 1.1-1.42.

Osano, S., 1989: Improvement of tropical cyclone analysis with satellite data. Part 2 of paper, Estimation of typhoon intensity from meteorological satellite data, for Economic and Social Commission for Asia and the Pacific \& WMO Typhoon Committee, 22nd session (30 Oct-6 Nov 1989), Tokyo, Japan. 6 pp.

Simiu, E., and R. H. Scanlon, 1978: Wind effects on structures. WileyInterscience, New York, NY. 458 pp.

Simpson, R. H., 1974: The Hurricane disaster potential scale. Weatherwise, 27, 109-186.

Webster, P. J., G. J. Holland, J. A. Curry, and H. R. Chang, 2005: Changes in tropical cyclone number, duration, and intensity in a warming environment. Science, 309, 1844-1846.

Yumoto, M., and T. Matuura, 2001: Interdecadal variability of tropical cyclone activity in the western North Pacific. J. Meteor. Soc. Japan, 79, 23-35.

Manuscript received 29 March 2006, accepted 12 June 2006

SOLA: http://www.jstage.jst.go.jp/browse/sola/ 